\title{
WSN-Life Enhancing Routing Algorithm
}

\author{
Pooja Nitin Vengurlekar \\ University of Pune \\ SKNCOE,Vadgaon(Bk), \\ Pune-41
}

\author{
Nalini.A.Mhetre \\ University of Pune \\ SKNCOE,Vadgaon(Bk), \\ Pune-41
}

\begin{abstract}
Wireless Sensor networks play an important role in the safety and security of industrial zones, forests etc. These networks are being used on a large scale for monitoring various environment conditions and also for agriculture across the globe. In India, from a past few years these networks are being used for Landslide Detection and monitoring. Wireless sensor Networks face a challenge of overcoming their short lifetime due to the usage of batteries in the sensor nodes. The energy Consumption in the sensor nodes is maximum during routing of the data.
\end{abstract}

The proposed algorithm aims at reducing this power consumption and improving routing of WSNs using the concept of fuzzy logic and A-star algorithm. Thus the proposed algorithm is combination of networking with artificial intelligence. The proposed algorithm uses mainly three factors minimum hop distance, remaining power of a node and minimum traffic load to make the routing decision. The performance of this algorithm is effective and could increase the overall life-time of an WSN by nearly 20$25 \%$.

\section{General Terms}

Wireless Sensor Networks Routing, Artificial Intelligence.

\section{Keywords}

Wireless sensor networks (WSNs), fuzzy logic, A-star algorithm, remaining power, traffic load

\section{INTRODUCTION}

Recent developments in sensor technology and in WSNs have lead many countries to use these networks for various purposes. From monitoring environmental conditions to battlefields, from agricultural fields to transportation of vegetables and fruits the sensors monitor the state and periodically report sensed data to the base station. Thousands of sensors are arranged to form a network across large fields to monitor and collect any malicious activity and send the data to the base station, which in turn is connected via internet to the user.

Figure 1 shows a WSN. The sensor nodes are arranged in an area. The sensor node closest to the target senses malicious activity and sends the sensed data to the nearest node, which in turn sends it to the nearest node on path of the sink. Finally the sink (base station) sends this information to the user via internet. Due to small communication range, sensor nodes transmit the data to the sink node via multiple hops. Thus, each sensor node acts as routing node for other nodes for other nodes.

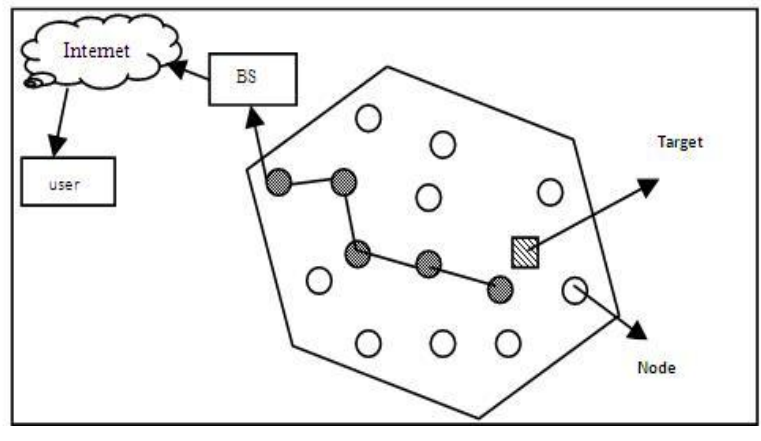

Fig 1: WSN Routing

Transmission of data. This leads to energy consumption of the node during routing. During routing the best path is chosen for transmission from source to sink. If the same path is chosen repeatedly then the nodes on this path will get drained fast. If energy consumption is not well man-aged, these nodes may eventually run out of power and this may lead to network failure. This paper proposes a method that tries to eradicate the problem of unbalanced energy consumption and tries to maximize the network lifetime of WSNs. We propose a new routing algorithm that uses fuzzy approach and A-star algorithm to select the optimal routing path from source to sink. The highest remaining battery power, minimum amount of traffic load and minimum number of hops are taken into consideration for selection of the next hop. Part 2 discusses the other works related to life enhancing routing. The design aspect of the proposed algorithm is discussed in Part 3 with help of mathematical model, Dynamic Programming etc. Part 4 discusses the results and part 5 is for conclusion and future work.

\section{RELATED WORK}

In traditional routing schemes of WSNs a node selects next hop based on minimum distance and also in order to prolong network lifetime. The current research thus focuses on optimal routing by taking into consideration the energy consumption. The work in [2] proposed to increase lifetime of WSN with the proposed algorithm, which considers sensor nodes in scheduling sets during routing of sensed data. However the nodes which are near the sink node always carry maximum traffic as they are always the part of the scheduling set, thus leading to depletion in their energy and finally failure.

In [4] the authors propose a method to increase the lifetime in terms of arranging the sensor nodes in disjoint sets. At a given point of time one set is active and the other one is in low power sleep mode. The sets become active successively. Thus if one of the sets fail others can be used. In [5] the authors propose a multiple sources multiple sinks model that optimizes network lifetime. MUSTER a heuristic proposed by 
the authors minimizes the number of nodes involved in many to many routing to balance their energy consumption. However, the method increases the overall cost of a WSN.

In [10] authors use two shortest path calculations to compute the routing path, however this method lacks path diversity. The work in [8] concentrates on the sleep and awake cycle of the nodes to increase lifetime. However it may lead to increased traffic on a certain path leading to congestion.

In [11] authors propose a methodology to minimize the hop stretch of a routing path in order to reduce energy cost of endto-end transmission. Similarly in [10] authors Deploy multiple sinks sensor nodes construct routes based on local topology information and the state information from sinks' broadcast messages. Sinks monitor their traffic load and adjust their own parameters to balance the traffic load in the network. However in both methods Due to the lack of path diversity nodes traversed by fixed routing paths may drain out quickly.

In [9] the opposite scenario is seen, the authors concentrate on the traffic load leading to ignorance to the remaining power in the batteries. The work in [6] uses the concept of clustering to increase the network lifetime. However it involves overhead of maintaining a cluster head and incase the cluster head fails then the recovery of transmitted data becomes difficult. In [7] the authors emphasize on path diversity however, this leads to ignorance of application load and remaining energy. In [2] the authors prove that routing in WSNs to increase network lifetime is a NP-Hard problem. They propose a heuristic method OML to increase network lifetime. In [3] the EEMLR algorithm uses a greedy approach to select the routing links. The energy cost of the links is taken into consideration to decide the routing path. However the heuristic used does not take into consideration the traffic load and may lead to congestion.

The proposed algorithm outperforms most of the papers cited in above paragraphs. It not only increases the lifetime of the network but also avoids congestion and increases routing speed. The set up of the proposed algorithm involves gathering of minimum distance and remaining energy from the nodes by the base station. The base station calculates the routing schedule and broadcasts it. A-star algorithm is applied to each node to find the optimal path. The fuzzy approach is used to find the value of cost of a node based on remaining energy and the traffic load.

\section{PROGRAMMER'S DESIGN}

The input to the program consists of the values of remaining energy and minimum hop of each sensor node from its neighbors. A-star algorithm applied for each node finds the minimum distance (path) of given node to sink. Thus the output of A-star algorithm will be minimum hop distances.

The fuzzy logic will be provided with RP (Remaining power) and Traffic Load (TL) values of each node. The output of this logic will be the Node cost i.e. the value of a node in terms of high or low. The final routing will utilize output of fuzzy logic and A-star algorithm to find the value of function $f(n)$ for each node. The node with highest $f(n)$ will be selected for routing path.
Table 3.1 Input and output at each stage

\begin{tabular}{|c|c|c|}
\hline Description & Input & Output \\
\hline A-star algorithm & $\begin{array}{c}\text { Distance between } \\
\text { nodes }\end{array}$ & Min hop $\forall \mathrm{n}$ \\
\hline Fuzzy Logic & RP,TL & node cost $\forall \mathrm{n}$ \\
\hline Final f(n) & $\begin{array}{c}\text { min hop and } \\
\mathrm{CN}(\mathrm{n}) \forall \mathrm{n}\end{array}$ & $\mathrm{f}(\mathrm{n})$ \\
\hline
\end{tabular}

\subsection{Mathematical Model \\ Terms used}

$\mathrm{RP}=$ Remaining power of a node i.e. battery power remaining in the node.

$\mathrm{TL}=$ Traffic load i.e. pending amount of traffic in node's queue. It also includes application traffic.

Minimum hop=Smallest path from source to sink (minimum hops required to reach sink)

For calculating the routing path we calculate the value $f(n)$ for each node using the formulae.

$\mathrm{f}(\mathrm{n})=\mathrm{CN}(\mathrm{n})+(1 / \mathrm{MH}(\mathrm{n}))$

The value of CN (n) is obtained from fuzzy approach using $\mathrm{RP}, \mathrm{TL}$. Whereas the value of MH (n) is calculated using Astar algorithm.

\subsubsection{Calculation of cost of node using fuzzy logic}

The value of $\mathrm{CN}(\mathrm{n})$ is calculated using fuzzy approach. In a fuzzy approach information is analyzed using fuzzy sets which are represented in linguistic manner. For e.g. low, high, moderate etc. If $\mathrm{Y}$ is a collection of objects denoted by $\mathrm{y}, \mathrm{Y}$ is called as universe of discourse and then fuzzy set $\mathrm{A}$ in $\mathrm{Y}$ is defined as follows

$$
\mathrm{A}=(\mathrm{y}, \mu \mathrm{A}(\mathrm{y}) / \mathrm{y} \epsilon \mathrm{Y}) \text {. }
$$

$\mu \mathrm{A}(\mathrm{y})$ is membership function of object $\mathrm{y}$ in $\mathrm{A}$. It de-fines degree of belongingness of each object in set A. The degree is usually a value in range [0 to 1$]$. A membership value is close to the value $1(\mu \mathrm{A}(\mathrm{y}) \rightarrow 1)$, it means that in-put y belongs to the set $\mathrm{A}$ with a high degree, whereas if $(\mu \mathrm{A}(\mathrm{y}) \rightarrow 0)$, indicates that set $\mathrm{A}$ does not suit input y very well. It uses IF-THEN rules that derive solution of the form IF antecedent(s) THEN consequent(s). Fuzzy sets and fuzzy rules form the knowledge base of a rule-based inference system. We use the values of $\mathrm{RP}$ and TL as antecedents to generate $\mathrm{CN}$ (cost of node) as consequent. AND operation is used to derive consequent result i.e. IF RP is High AND TL is low then cost of node $=\mathrm{HIGH}$.

\subsubsection{Finding $M H(n)$ using A-star Algorithm}

A-star algorithm works on the formulae $f(n)=g(n)+h(n)$ $g(n)=$ actual cost from initial node to node $n . h(n)=$ heuristic function, estimate of optimal path cost from $n$ to target node. A-star creates tree structure to search optimal routing path from any node $\mathrm{x}$ to the base station. In our case using equation (1) we find $\mathrm{MH}(\mathrm{n})$ using A-star algorithm.

\subsection{Dynamic Programming and Serialization}

The basic principle of this paper is to find routing paths such that routing is optimal as well as energy is con-served for all sensor nodes. In the proposed method we make use of solving the routing path problem by considering three factors RP, TL and $\mathrm{MH}$. The problem of finding the MH for each node $\mathrm{n}$ is solved using A-star algorithm. Whereas the cost of a node found using fuzzy logic takes into consideration TL and RP. 
Thus the problem gets divided into two parts 1) Find minimum hop and 2) find Cost of node, the ultimate value of a node $\mathrm{n}$ is as given in equation 1 i.e. obtained by combining both the solutions. Further in A-star algorithm, the nodes are divided into visited and unvisited sets and the optimal path is kept in the CLOSED list. Each node is visited once and its distance from source node is checked. A tree structure is maintained to keep track of visited and unvisited nodes. A-star is a combination of Breadth first search and depth first search.

\subsection{Data independence and Data Flow} architecture

Data table will be used to store the Rule base of the fuzzy logic. The table will be updated on every result derived after fuzzy logic is executed.

\subsection{Multiplexer Logic}

The proposed algorithm consists of mainly two computations finding minimum hop and finding cost of a node. These computations are independent of each other, thus the A-star algorithm and fuzzy logic can be processed concurrently using multi-threading. A star algorithm is applied for each node to find its minimum hop to sink. Thus if there are $\mathrm{n}$ nodes then for each of these $\mathrm{n}$ nodes the algorithm will check remaining $\mathrm{n}-1$ nodes to find minimum path. The computation might thus take $\mathrm{O}\left(\mathrm{n}^{2}\right)$. To reduce this time we can make use of multithreading at sink node. If multiple sink nodes are used then the computation time can be reduced further as the task of minimum hop calculation for half nodes can be done at other sink. However multiple sink nodes face challenges of increase cost of network and co-ordination amongst the sink nodes.

\subsection{Turing Machine}

Figure 2 shows state transition diagram of a sensor node in an WSN The sensor nodes are in active and passive (sleep) node after fixed interval. The sensors sense data on being active. They process the data and send it to sink node via routing path. After sending completes the nodes go back to passive state. Similarly, when in active state, after regular interval the sensor node send RP and TL information to the Base station. On reception of routing schedule the routing table is updated and the sensor goes back to active state. Fig 3 on next page shows the states at the Base station end. The BS is in the listen state, if it receives TL and RP information from nodes it processes this data using a star algorithm and fuzzy logic.

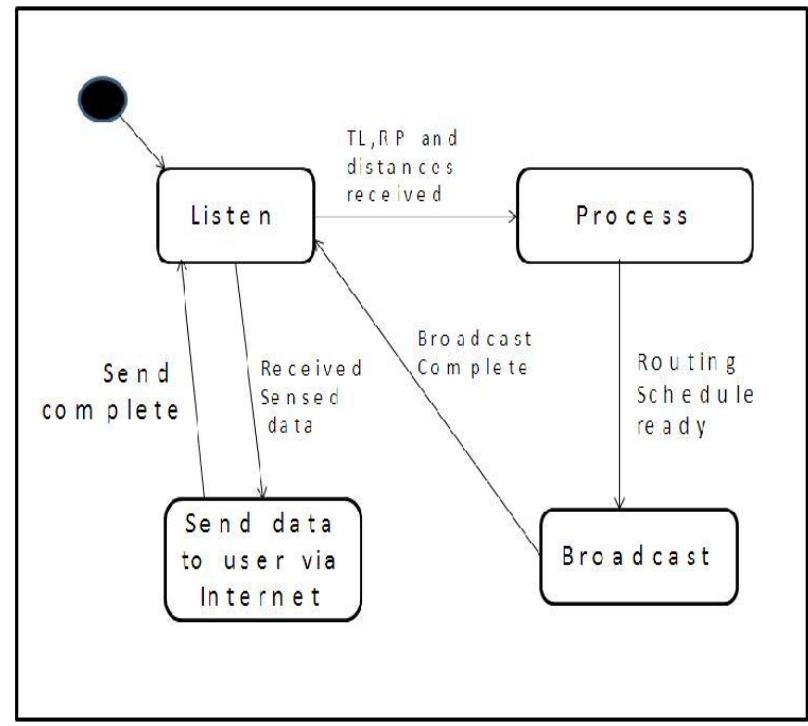

Fig 2 : State diagram for sensor nodes

logic to produce the routing schedule, which it broadcasts to all the nodes. In turn if it receives sensed data from sensor nodes, it sends it to user/operator via internet. After broadcasting or sending data the BS goes back to Listen mode.

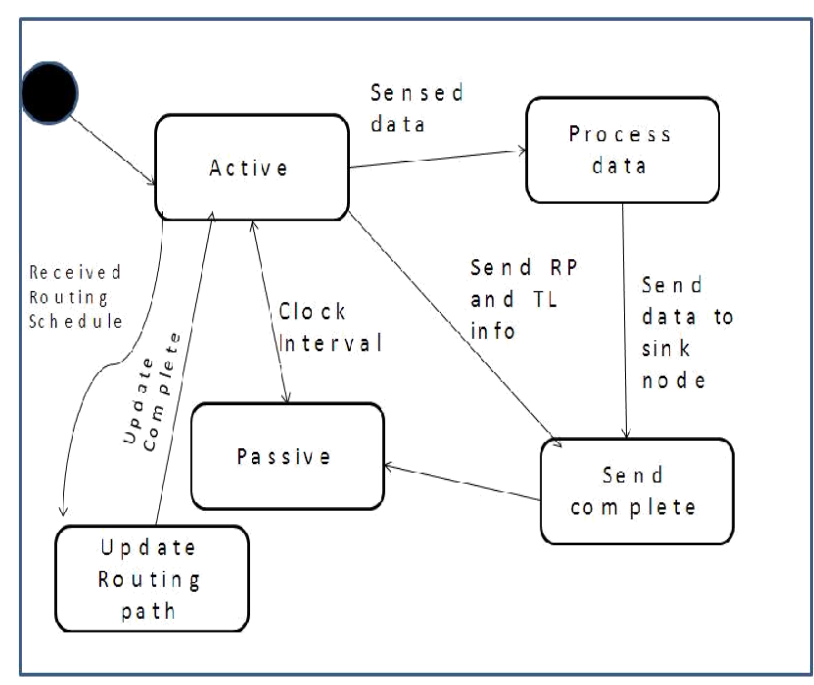

Fig 3 : State diagram for base station

\section{RESULTS AND DISCUSSION}

The proposed algorithm is expected to increase the life-time of the WSN by nearly $20-25 \%$. It can also produce lower end to end delay in data transmission from nodes to sink.

\section{CONCLUSION}

The challenge in routing of WSN lies in not just lower end to end delay, but also saving energy of nodes. In the current scenario, the proposed algorithm can outperform many existing routing algorithms in terms of efficiency as well as lifetime enhancement. The algorithm not only manages routing optimally but also avoids network partitions caused due to prolonged use of same path for routing. The proposed algorithm can produce better speed up if used in multithreaded environment and if multiple sink nodes are used. 


\section{ACKNOWLEDGMENTS}

We would like to thank the experts who have contributed towards development of this article.

\section{REFERENCE}

[1] Imad S. AlShawi, Lianshan Yan, Wei Pan, and Bin Luo,2012 Lifetime Enhancement in Wireless Sensor Networks using Fuzzy Approach and A-Star Algorithm

[2] Yuping Dong,Hwa Chang,Zhongjian Zou,Sai Tang,"An Energy Conserving Routing Algorithm for Wireless Sensor Networks" International Journal of Future Generation Communication and NetworkingVol. 4, No. 1, March 2011

[3] Sourabh Jain, Praveen Kaushik, Jyoti Singhai "ENERGY EFFICIENT MAXIMUM LIFETIME ROUTING FOR WIRELESS SENSOR NETWORK", International Journal Of Advanced Smart Sensor Network Systems ( IJASSN ), Vol 2, Jan 2012

[4] MIHAELA CARDEI, DING-ZHU DU " Improving Wireless Sensor Network Lifetime through Power Aware Organization", Wireless Networks 11, 333-340, 2005 springer.

[5] Luca Mottola, Member, IEEE ComputerSociety, and Gian Pietro Picco , "MUSTER: Adaptive Energy-Aware Multisink Routing in Wireless Sensor Networks"

[6] Arash Ghorbannia Delavar, Somayeh Shamsi Nafiseh Mirkazemi, Javad Artin "SLGC: A NEW CLUSTER ROUTING ALGORITHM IN WIRELESS SENSOR NETWORK FOR DECREASE ENERGY CONSUMPTION", International Journal of Computer Science, Engineering and Applications (IJCSEA) Vol.2, No.3, June 2012

[7] Joon-Woo Lee , Ju-Jang Lee "Ant-Colony Based Scheduling Algorithm for Energy-Efficient Coverage of WSN", IEEE SENSORS JOURNAL, VOL. 12, NO. 10, OCTOBER 2012
[8] Joongseok Park and Sartaj Sahi,"An Online Heuristic for Maximum Lifetime Routing in Wireless Sensor Networks",IEEE TRANSACTIONS ON COMPUTERS, VOL. 55, NO. 8, AUGUST 2006

[9] M. R. Minhas, S. Gopalakrishnan, and V. C. M. Leung, "An online multipath routing algorithm for maximizing lifetime in wireless sensor networks," in Proc. IEEE Inform. Technol. New Generat. 6th Int. Conf., Apr. 2009

[10] C. Wu, R. Yuan, and H. Zhou, "A novel load balanced and lifetime maximization routing protocol in wireless sensor networks," in Proc.IEEE Vehicular Technol. Conf. VTC Spring, May 2008

[11] M. J. Tsai, H. Y. Yang, and W. Q. Huang, "Axis-based virtual coordinate assignment protocol and deliveryguaranteed routing protocol in wireless sensor networks," in Proc. IEEE INFOCOM 26th Int. Conf. Comput. Commun., May 2007

[12] Cunqing Hua and Tak-Shing Peter Yum,"Optimal Routing and Data Aggregation for Maximizing Lifetime of Wireless Sensor Networks "IEEE/ACM TRANSACTIONS ON NETWORKING, VOL. 16, NO. 4, AUGUST 2008

[13] S.Anandamurugan,C.Venkatesh"Increasing the Lifetime of Wireless Sensor Networks by using AR (Aggregation Routing) Algorithm," in Proc. IEEE Global Telecommun. Conf., Nov. 2007, pp. 710714.

[14] S. Y. Chiang and J. L. Wang, "Routing analysis using fuzzy logic systems in wireless sensor networks," Lecture Notes Comput. Sci., vol. 5178, pp. 2008.

[15] K. M. Rana and M. A. Zaveri, "ASEER: A routing method to extend life of two-tiered wireless sensor network," Int. J. Adv. Smart Sensor Netw Syst., vol. 11, no. 2, pp. 1U16, Oct. 2011.

[16] W. Dargie and C. Poellabauer, "Network layer" ,in Fundamental of Wireless Sensor Networks Theory and Practice. New York: Wiley, 2010, pp. 